Emmanuelle Chapron, «La culture scolaire au village : la place du livre », dans Frédéric Boutoulle, Stéphane Gomis (dir.), Cultures villageoises au Moyen Âge et à l'époque moderne, Actes des XXXVII ${ }^{\mathrm{e}}$ Journées internationales d'histoire de l'abbaye de Flaran, 9 et 10 octobre 2015, Toulouse, Presses universitaires du Midi, 2017, p. 39-58.

Mots clés : école, village, livres, France, XVIII ${ }^{\mathrm{e}}$ siècle.

Résumé :

Les livres remis aux enfants qui allaient à l'école, dans la France du XVIII siècle, s'y trouvaient-ils du fait de la « fantaisie des parents », comme le suggèrent les correspondants de l'abbé Grégoire, sous la Révolution ? Il s'agit de prendre au sérieux cette question, en mettant en évidence comment jouent autour de ces livres un ensemble de régulations sociales, matérielles et intellectuelles qui contrebalancent l'arbitraire apparent de leur sélection. Le contrôle de l'Église sur les petites écoles, l'économie du livre au village, l'appropriation de la structure scolaire par les populations, les pratiques pédagogiques en usage, dessinent des corpus qui ont leur logique propre, qui ne coïncident qu'en partie mais dont l'ensemble présente une véritable cohérence, au point de pouvoir parler d'un « ordre scolaire des livres ».

\title{
La culture scolaire au village : la place du livre
}

\author{
Emmanuelle Chapron \\ Aix Marseille Université, CNRS, UMR 7303, Aix-en-Provence, France
}

À l'automne 1790, l'abbé Grégoire soumet à ses concitoyens une enquête devenue célèbre $^{1}$. Entre les questions relatives aux patois et celles qui concernent les superstitions, quatre s'intéressent aux petites écoles. À cet endroit charnière, elles reflètent la confiance que les hommes de la Révolution, Grégoire en tête, ont placée dans l'enseignement comme moyen de modeler l'homme nouveau. Les réponses au questionnaire doivent permettre d'identifier les obstacles à lever pour atteindre cet objectif: inégalités territoriales dans l'implantation des écoles, persistance de l'usage du patois dans les interactions entre le maître et l'élève, disparité des outils et des méthodes pédagogiques. La question 31 est précisément la suivante : «Dans les écoles de la campagne l'enseignement se fait-il en français ? Les livres y sont-ils uniformes? ». Les réponses, on s'en doute, ont une tonalité très négative, révélant le sentiment général des élites à l'égard des petites écoles rurales. L'usage étant que les enfants apportent les livres de chez eux, l'école ne peut être que le royaume de la « fantaisie des parents », comme l'exprime l'abbé Andriès, de Berques en Flandre-Maritime ${ }^{2}$. Que la pratique des paysans soit régie par le hasard, il ne pouvait guère en être autrement pour les correspondants lettrés de l'abbé Grégoire, chez qui l'on retrouve les schémas dominants de l'imaginaire du peuple au XVIII ${ }^{\mathrm{e}}$ siècle, doublés d'un souci de légitimation face à leur interlocuteur parisien et, parfois, d'un intérêt sincère et désolé pour l'éducation

\footnotetext{
${ }^{1}$ M. de Certeau, D. Julia, J. Revel, Une politique de la langue. La Révolution française et les patois : l'enquête de Grégoire, Paris, 1975 ( $3^{\mathrm{e}}$ éd., 2002). Une partie des réponses a été publiée par A. Gazier, Lettres à Grégoire sur les patois de la France (1790-1794). Documents inédits sur la langue, les mours et l'état des esprits dans les diverses régions de la France au début de la Révolution, Paris, 1880. Une partie se trouve rassemblée dans le ms. B.N., NAF 2798.

${ }^{2}$ Ibid., fol. 21 .
} 
populaire $^{3}$. Même lorsque les correspondants répondent à la question par l'affirmative (ce qui est quand même le cas dans plus de la moitié des réponses), l'uniformité des livres qu'ils mentionnent ne relève pas d'une volonté de principe, mais bien plutôt de la routine et de la pauvreté. «Les livres sont ordinairement uniformes, le catéchisme, un syllabaire, le Chemin du Ciel voilà les seuls livres qu'on emploie », signale le correspondant bressan Jean-Baptiste de Cherval ${ }^{4}$. L'école paysanne reste dans tous les cas un monde scolaire mal réglé ou réglé par défaut, un monde où le choix des supports de l'apprentissage résulte de l'arbitraire, de l'indigence, de ce que l'Église laisse dans les campagnes. Tout semble susceptible de pouvoir servir, un jour ou l'autre, à l'enfant allant à l'école de campagne, au point qu'il faudrait parler de « livres utilisés dans les classes », plutôt que de « livres de classe ».

S'arrêter à ce constat, c'est s'interdire de comprendre ce que la petite école peut apporter à la culture de l'écrit dans les milieux populaires ruraux. Certes, le choix des livres utilisés dans les écoles est forcément tributaire des ressources comptées de la librairie rurale et de l'intense politique d'acculturation par l'écrit menée par l'Église au XVIII ${ }^{\mathrm{e}}$ siècle. Mais du fait même des fonctions religieuses et sociales dont elle est investie, l'école n'absorbe pas indifféremment toutes sortes d'imprimés. Par ailleurs, quand bien même les livres employés pour l'enseignement ne lui seraient pas spécifiques, l'école ne peut être un simple espace de répétition et de reproduction des pratiques qui lui sont extérieures (le chant de l'église, le catéchisme dominical, les lectures pieuses ou récréatives faites en famille). De par son organisation, si rudimentaire soit-elle (un maître rassemblant des élèves dans un même endroit pour les enseigner), la scolarisation s'accompagne de manières propres de manipuler le livre et d'appréhender l'écrit.

Il s'agit donc de prendre au sérieux la question des ouvrages utilisés dans les petites écoles, en mettant en évidence comment jouent autour d'eux un ensemble de régulations sociales, matérielles et intellectuelles qui contrebalancent l'arbitraire apparent de leur sélection. Le contrôle de l'Église sur les petites écoles, l'économie du livre au village, l'appropriation de la structure scolaire par les populations, les pratiques pédagogiques en usage, dessinent des corpus qui ont leur logique propre, qui ne coïncident qu'en partie mais dont l'ensemble présente une véritable cohérence, au point de pouvoir parler d'un «ordre scolaire des livres $\gg$.

\section{Le livre d'école sous le contrôle de l'Église}

L'enjeu que constitue le façonnement de l'enfance chrétienne explique l'attention portée par l'Église aux ouvrages utilisés en classe. Au XVIII ${ }^{\mathrm{e}}$ siècle, alors que la pratique religieuse est de plus en plus médiatisée par l'écrit, l'école constitue un lieu d'acculturation à la manipulation des ouvrages de dévotion produits en quantités colossales par les imprimeurs du royaume, livres d'office qui permettent de suivre le service divin, ou livres de piété qui nourrissent la pratique personnelle. Cette préoccupation se traduit d'abord par des mesures défensives ; car tant que les enfants viennent à l'école munis de leurs propres instruments de travail, il y a souvent à craindre de ce qu'ils apportent. Curés et maîtres sont chargés de faire la chasse aux mauvais livres, c'est-à-dire aux livres hétérodoxes et à la littérature récréative, mais également, de manière plus sensible quoique plus géographiquement différenciée au cours du siècle, aux livres jansénistes.

\section{Livres périlleux}

\footnotetext{
3 D. Cohen, La nature du peuple: les formes de l'imaginaire social, XVIII ${ }^{e}-X X I^{e}$ siècle, Seyssel, 2010. R. Chartier, « Lectures paysannes. La bibliothèque de l'enquête Grégoire », XVIII siècle, 18, 1986, p. 45-64.

${ }^{4}$ B.N., NAF 2798, fol. 56v. Autres réponses similaires dans le district de Château-Thierry (ibid., fol. 41) et dans le Poitou (A. Gazier, Lettres à Grégoire..., p. 278).
} 
La contamination des écoliers catholiques par une circulation incontrôlée des ouvrages protestants est une crainte ancienne. C'est d'abord la hantise de l'hétérodoxie qui motive, au XVII ${ }^{\mathrm{e}}$ siècle, l'intérêt porté par les évêques aux livres utilisés dans les classes. Lors de son premier synode en avril 1638, l'évêque de Cahors, Alain de Solminihac, astreint les recteurs des paroisses à donner "advis des maistres d'eschole qui sont ez paroisses des champs", mais aussi «de quels livres ils enseignent $»^{5}$. À la même époque, l'évêque de Châlons-surMarne, Félix Vialart, s'inquiète des éditions réformées de la Bible en français, qui pourraient se trouver encore dans son diocèse (la présence protestante y a été forte) et être mises, en toute bonne foi, entre les mains des enfants allant à l'école ${ }^{6}$. La question est toujours sensible au début du XVIII ${ }^{\mathrm{e}}$ siècle. Le programme de scolarisation forcée mis en place par la monarchie à partir des années $1660 \mathrm{a}$, en effet, paradoxalement participé à redynamiser le marché clandestin du livre protestant, que l'on voit se reconstituer à partir des années 1710 . En 1731, l'intendant de Montauban écrit à M. de Saint-Florentin qu' « il ne résultait autre chose de ces écoles forcées, sinon que les parents se donnaient plus de soin pour avoir des livres de calvinisme et prenaient plus à tâche de s'instruire eux-mêmes dans cette religion, pour pouvoir au retour des écoles détruire plus efficacement et plus savamment les teintures qu'elles auraient pu donner $»^{7}$. C'est à la même époque que l'évêque de Mende, Choiseul de Beaupré fait explicitement figurer au nombre des obligations des maîtres d'école, dans les brevets de nomination, celle de saisir les livres hérétiques pour les remettre au curé ${ }^{8}$. Envisage-t-il réellement que les enfants puissent venir à l'école avec des psaumes et des Nouveaux Testaments tirés des coffres domestiques? On imagine mal que les nouveaux convertis, appelés par leurs prédicants à cacher et à conserver précieusement leurs Bibles et leurs livres de piété, en aient muni leurs enfants ${ }^{9}$. Là où la présence protestante se raréfie, il est en revanche possible que des ouvrages réformés aient échappé à la destruction et continuent à être utilisés par souci d'économie. On retrouve bien dans l'église de MontignyMontfort, dans le diocèse de Langres, en 1740, deux coffres qu'on dit renfermer " plusieurs livres et manuscrits contraires à la religion et qui favorisent le calvinisme ", mélangés à des affaires appartenant à la fabrique, et que l'évêque envoie déminer avec le plus grand $\operatorname{soin}^{10}$.

Dès le XVII ${ }^{\mathrm{e}}$ siècle, les lectures profanes constituent la seconde cible des règlements épiscopaux. Présents dès cette époque dans les milieux populaires urbains, les récits burlesques, les recueils de contes et les livres de chansons se répandent dans les campagnes dans la première moitié du XVIII ${ }^{\mathrm{e}}$ siècle, à la faveur de l'alphabétisation et de l'activité des colporteurs $^{11}$. À ces lectures, les ordonnances épiscopales tentent de fermer la voie de l'école. En 1678, l'évêque d'Angers ordonne aux maîtres de «bannir des petites écoles les livres de fables, les romans et toutes sortes de livres profanes et ridicules dont on se sert pour

\footnotetext{
5 G. Astoul, Les chemins du savoir en Quercy et Rouergue à l'Époque moderne. Alphabétisation et apprentissages culturels, Toulouse, 1999, p. 63.

${ }^{6}$ «Règlements sur plusieurs points de la discipline ecclésiastique » [1662], Statuts, ordonnances, mandemens, reglemens, et lettres pastorales, imprimez par ordre de Louis-Antoine de Noailles, évêque comte de Chaalons, Châlons, 1693, p. 42-43.

${ }^{7}$ F. Balestie, Le Montalbanais, des dragonnades au Réveil, Montauban, 1971, p. 76.

${ }^{8}$ M. Laget, «Petites écoles en Languedoc au XVIII ${ }^{\mathrm{e}}$ siècle », Annales Économies, Sociétés, Civilisations, 26, 1971, p. 1398-1418; ici, p. 1410.

${ }^{9}$ C. Bernat, «Armer les esprits. Instruction et combat dans la littérature pastorale réformée du premier Désert (1685-1745) », Y. Krumenacker, B. Nogues (éd.), Protestantisme et éducation dans la France moderne, Lyon, 2014, p. 197-228, notamment p. 218.

${ }^{10}$ A.D. Haute-Marne, G 971, p. 519.

${ }^{11}$ J.-L. Marais, "Littérature et culture "populaires" aux XVII ${ }^{\mathrm{e}}$ et XVIII ${ }^{\mathrm{e}}$ siècles », Annales de Bretagne et des pays de l'Ouest, 87, 1980, p. 65-105 ; R. Chartier, «Livres bleus et lectures populaires », R. Chartier, H.J. Martin (éd.), Histoire de l'édition française, t. 2, Le livre triomphant, 1660-1830, Paris, 1984, p. 498-511.
} 
commencer à leur apprendre à lire $»^{12}$. La mention rappelle que les imprimés de large diffusion comme ceux des Bibliothèques bleues de Troyes, de Rouen ou de Limoges ont pu constituer un des instruments de l'alphabétisation domestique. Que ces petits livres aient été ou non utilisés dans les écoles, à l'insu des autorités ecclésiastiques, est en revanche une question délicate à trancher. Les ouvrages utilisés dans les écoles ne font pas l'objet de questions particulières dans les formulaires de visites pastorales, même s'ils peuvent être mentionnés à l'occasion. À Harfleur et à Montivilliers, deux villages visités par l'archevêque de Rouen en 1660, la maîtresse Jeanne L'Allouette et l'écrivain Jean Boutevillain se voient rappeler les « précautions à prendre contre les livres profanes et apocryphes ou défendus $»^{13}$. On ne sait pas quelles observations ont motivé l'avertissement; mais il n'était sans doute pas jugé inutile dans un diocèse marqué par une production massive et précoce d'ouvrages protestants et de livrets à bon marché, y compris de textes religieux que les censeurs qualifient d' « apocryphes », c'est-à-dire d'absurdes et de superstitieux ${ }^{14}$. En 1691, lorsque l'archevêque de Bordeaux, Louis d'Anglure de Bourlement, organise une inspection complète du diocèse, le délégué qui visite la paroisse de Gujan s'informe auprès du régent « quels livres ses écoliers lisaient à l'école». Mais la mention est isolée dans la source, et la réponse apportée est d'ailleurs tout à fait satisfaisante ${ }^{15}$.

$\mathrm{Au}$ cours du XVIII ${ }^{\mathrm{e}}$ siècle, la question des livres utilisés dans les écoles paraît secondaire dans les préoccupations épiscopales, soit que la menace semble s'être éloignée (ouvrages réformés), soit qu'elle apparaisse encore peu tangible dans les campagnes (livrets de colportage). A contrario, la capacité de l'épiscopat français à réactiver la surveillance des écoles, au moment où une partie des évêques s'inquiète de la diffusion des imprimés jansénistes, montre combien le risque n'a jamais été perdu de vue. De façon significative, la seule enquête épiscopale du XVIII ${ }^{\mathrm{e}}$ siècle qui comprenne des questions systématiques sur les ouvrages utilisés pour l'instruction scolaire est celle que conduit en 1777-1778 l'évêque d'Auxerre, Baptiste-Marie Champion de Cicé, confronté à la persistance de l'influence janséniste après l'épiscopat de Charles de Caylus ${ }^{16}$. Les conflits liés aux livres utilisés dans les écoles jansénistes, ou aux livres jansénistes utilisés dans les écoles, ne sont de fait pas rares. Leur fréquence tient à l'attitude très volontariste des élites jansénistes en matière de fournitures scolaires : on sait l'importance de l'écrit dans la pastorale du parti, qui passe par une large diffusion de livres de piété, de brochures et de Nouveaux Testaments. Dans les diocèses repris en main par des évêques antijansénistes, la lutte passe par le réarmement de tous les dispositifs de surveillance, de dénonciation et d'enquête sur le terrain. Les mesures prises dans le diocèse de Langres par l'évêque Gilbert de Montmorin montrent la petite école rurale comme l'un des lieux où l'on craint de voir s'accrocher l' "hérésie nouvelle ». En 1740, les doyens et vice-doyens ruraux sont spécialement chargés de s'informer «si on distribue dans leurs doyennés des livres dangereux, soit par rapport à la foi, soit par rapport aux mœurs, soit dans les écoles, soit autrement $\gg{ }^{17}$. L'année suivante, une ordonnance

\footnotetext{
${ }^{12}$ Statuts du diocèse d'Angers, 1680, p. 768.

${ }^{13}$ C. de Robillard, Recherches sur l'instruction publique dans le diocèse de Rouen avant 1789, Évreux, 1872 , p. 373,375 .

${ }^{14}$ J.-D. Mellot, L'édition rouennaise et ses marchés (vers 1600-vers 1730). Dynamisme provincial et centralisme parisien, Paris, 1998, p. 495.

${ }^{15}$ L. Bergès, « Renouveler l'histoire de l'éducation sous l'Ancien Régime à Bordeaux et en Guyenne : sources documentaires locales et pistes de recherche », Revue historique de Bordeaux et du département de la Gironde, 13-14, 2008, p. 19-43.

${ }^{16}$ A.D. Yonne, G 1668. D. Dinet, «Une déchristianisation provinciale au XVIII ${ }^{\mathrm{e}}$ siècle : le diocèse d'Auxerre », Histoire, économie et société, 10, 1991, p. 467-489.

${ }^{17}$ A.D. Haute-Marne, G 971, 20 avril 1740, p. 301-305. D. Dinet, « Administration épiscopale et vie religieuse au milieu du XVIII ${ }^{\mathrm{e}}$ siècle : le Bureau pour le gouvernement du diocèse de Langres de Gilbert de Montmorin », Revue d'histoire ecclésiastique, 78, 1983, p. 721-774.
} 
épiscopale ordonne aux curés « de nous donner des listes les plus exactes qu'ils pourront des livres qui sont en usage dans les écoles, ou qui sont répandus dans les maisons particulières $»^{18}$. Une telle volonté de percer le for domestique de la lecture, en combinant l'esprit inquisitorial et la culture administrative de la liste, n'a guère de précédent. Si rien ne confirme que ces listes aient été réellement dressées, la surveillance renforcée sur les paroisses débouche sur un certain nombre de signalements : en 1747, l'évêque diligente une enquête sur le maître d'école de Chessy, «accusé d'enseigner à lire dans les Réflexions morales du P. Quesnel et d'enseigner le catéchisme du diocèse d'Auxerre ${ }^{19}$. En dehors des diocèses tenus par ces évêques zélés, il n'est pas certain que la surveillance sur les livres des écoles ait été très serrée. Dans ces diocèses mêmes, la manière dont le monde clérical a relayé au niveau paroissial ces préoccupations disciplinaires pose d'ailleurs question. La même chose vaut pour les populations qui peuvent s'opposer à cette censure pour des raisons multiples: conviction doctrinale, attachement à un catéchisme transmis sur plusieurs générations, préoccupations économiques. Tant que la fourniture des livres reste à la charge financière des familles, la censure paroissiale est délicate à mettre en œuvre.

\section{Donner à lire}

En parallèle des mesures défensives, les évêques du royaume travaillent à promouvoir des pratiques de bonne lecture auprès des fidèles. Cette politique correspond à une mutation de grande ampleur de l'attitude de l'Église à l'égard du livre et de la lecture, désormais considérés comme des vecteurs de la bonne foi. Dès les dernières décennies du XVII ${ }^{\mathrm{e}}$ siècle, l'épiscopat se lance dans la production d'instruments à l'usage des classes. La production la plus emblématique est celle des catéchismes «moyens", ceux que l'on utilise dans les écoles, dont la production connaît un décollage spectaculaire à partir des années $1670^{20}$. Leur diffusion est d'autant plus grande qu'ils sont vendus à des prix modiques, 6 à 10 sols à la fin du siècle, ce qui les place au même niveau que les livrets de colportage. Si l'on considère que le tarif pour l'apprentissage de la lecture est en moyenne de 5 sous par mois et de 10 sous pour l'écriture, il n'en coûte pas plus de l'équivalent d'un « treizième mois » d'écolage pour acheter un catéchisme, et les distributions gratuites ne sont d'ailleurs pas rares.

Le cadre scolaire induit des pratiques qui ne sont pas absolument comparables à celles de la catéchèse ecclésiale ${ }^{21}$. Sans perdre de vue l'impératif de faire accéder l'enfant aux vérités de la foi, la catéchèse scolaire est arrimée à l'apprentissage de la lecture. Dans beaucoup de diocèses, le catéchisme doit constituer le premier livre de lecture suivie, après l'étape du syllabaire $^{22}$. Cette position dans le cursus des apprentissages a parfois conduit les imprimeurs à faire glisser le catéchisme vers les outils de la première alphabétisation : on en trouve une illustration dans le Catéchisme du diocèse de Meaux (Paris, La Caille, 1674), où un syllabaire a été imprimé au revers de la page de titre, probablement à l'initiative de l'imprimeur. Par ailleurs, alors que l'usage de l'idiome local est assez répandu dans la catéchèse paroissiale, la question de la langue peut se poser autrement dans le cadre scolaire. En 1752, les habitants de

\footnotetext{
${ }^{18}$ A.D. Haute-Marne, G 972, 13 avril 1741, p. 30.

${ }^{19}$ A.D. Haute-Marne, G 974, p. 44.

${ }^{20} \mathrm{La}$ même remarque vaut pour l'ensemble de la production catéchétique. A. Monaque (dir.), Catéchismes diocésains de la France d'Ancien Régime, Paris, 2001.

${ }^{21}$ J.-R. Armogathe, «Les catéchismes et l'enseignement populaire en France au XVIII ${ }^{\mathrm{e}}$ siècle », Images du peuple au XVIII e siècle, Paris, 1973, p. 103-121 ; M. Venard, "Catéchisme à l'église ou catéchisme à l'école ? Un choix ecclésiologique et social dans la France des $\mathrm{XVI}^{\mathrm{e}}$ et $\mathrm{XVII}{ }^{\mathrm{e}}$ siècles », Enseigner le catéchisme, autorité et institutions, $X V I^{e}-X X^{e}$ siècles, Québec-Paris, 1997, p. 57-72 ; J. de Viguerie, "Les catéchismes enseignés en France au XVIII siècle. Première approche », Revue d'histoire de l'Église de France, 82, 1996, p. 85-108.

${ }^{22}$ Ordonnances synodales du diocèse de Bordeaux, Bordeaux, 1728. Voir également R. Grevet, École, pouvoirs et société : fin XVII siècle-1815 Artois, Boulonnais-Pas-de-Calais, Villeneuve-d'Ascq, 1991, p. 109.
} 
Capbreton, dans le diocèse de Dax, reprochent à leur évêque d'avoir ordonné aux curés, vicaires et «même aux maîtres d'école »d'enseigner le catéchisme en basque ${ }^{23}$. La querelle porte bien sur le cadre scolaire, où se cristallisent les intérêts divergents des bourgeois de la ville, intéressés à maintenir les distinctions linguistiques et à consolider chez leurs enfants une compétence qui vaut capital social, et le prélat, avant tout préoccupé de la bonne compréhension du message catéchétique dans une population principalement composée de pêcheurs et de vignerons patoisants. Il n'est pas évident que le problème se soit posé partout dans les mêmes termes, comme en Bretagne où même la catéchèse scolaire a pu être pratiquée en breton ${ }^{24}$.

Le répertoire élaboré dans les diocèses comprend également une large gamme d'heures et de psautiers "à l'usage des écoles », ainsi que des histoires bibliques infantilisées, qui commencent à paraître dans les années $1680^{25}$. Toutefois, il ne faut sans doute pas exagérer l'ampleur de l'effort d'adaptation fait par les autorités ecclésiastiques. À l'exception de quelques titres vraiment dédiés, les ouvrages recommandés par les ordonnances épiscopales puisent très largement dans le fonds commun de la littérature de dévotion. Jusqu'à la fin de l'Ancien Régime, l'école constitue un débouché essentiel pour les ouvrages de piété produits par les imprimeurs du royaume pour le tout-venant des fidèles. Il est même probable que le cadre scolaire ait offert une seconde vie à certains titres passés de mode auprès d'un public adulte. Il est ainsi frappant que tous les témoignages d'usage rassemblés par François Lebrun à propos des Sept trompettes pour réveiller les pécheurs - un grand succès de librairie depuis sa première édition à Bergame en 1612 - concernent les petites écoles ${ }^{26}$. Malgré tout, à des degrés divers selon les diocèses, c'est toute une gamme d'instruments scolaires qui est mise en place à l'initiative des évêques. Certes, toutes ces publications visent à renforcer l'école dans son rôle d'antichambre de l'église : c'est là que l'enfant apprend à entonner les chants, à répondre à la messe, à approfondir sa foi par la lecture des ouvrages de piété. Les catéchismes, les petites heures et les Bibles d'enfants circulent de l'église à l'école. Mais en infantilisant les instruments traditionnels de la piété chrétienne, l’Église participe à dessiner l'école comme le lieu d'une communauté de lecteurs, dont les expériences partagées s'adossent à des supports spécifiques.

\section{La « fantaisie des familles »}

Ces injonctions ecclésiastiques se heurtent à une réalité matérielle indépassable : dans la grande majorité des cas, c'est à l'élève d'apporter de chez lui les livres dont il se servira. Voilà le maître obligé de composer avec le bon vouloir des parents et tributaire des ressources domestiques. Mais avait-on seulement des livres à donner aux enfants ? Quels genres de livres pouvait-on leur confier pour aller à l'école?

\section{Un livre à soi ?}

\footnotetext{
${ }^{23}$ Serrurier, L’instruction primaire dans la région des Pyrénées occidentales, spécialement en Béarn, 1385-1789, Pau, 1874, p. 8-10.

${ }^{24}$ M. Lagrée (éd.), Les parlers de la foi. Religion et langues régionales, Rennes, 1995.

${ }^{25}$ Voir par exemple l'Instruction sur les petites écoles du diocèse de Mirepoix, Toulouse, 1699, contenant une histoire abrégée de l'Écriture sainte et de l'Évangile, et le Livre à l'usage des écoles du diocèse d'Amiens, où sont contenues les principales actions de la Vie de Notre Seigneur Jésus Christ, pour être enseigné après le catéchisme, Amiens, 1708.

${ }^{26} \mathrm{~F}$. Lebrun, «Un grand succès de la littérature religieuse aux XVII ${ }^{\mathrm{e}}$ et XVIII siècles : "Les sept trompettes pour réveiller les pécheurs" ", D. Turrel (éd.), Regards sur les sociétés modernes (XVI ${ }^{e}$-XVIII ${ }^{e}$ siècle). Mélanges offerts à Claude Petitfrère, Tours, 1997, p. 105-113.
} 
$\mathrm{Au} \mathrm{XVIII}^{\mathrm{e}}$ siècle encore, l'achat d'un livre est un geste peu banal pour la plus grande partie de la population française. Confier un livre à un enfant n'est donc pas une chose anodine, d'autant que rien n'assure que l'ouvrage reviendra en bon état, ou même qu'il reviendra tout court. On le munira probablement de brochures peu coûteuses, d'ouvrages inutilisés, avant que ses progrès et les espoirs que l'on place en lui ne justifient éventuellement d'autres investissements. Si l'on puisait d'abord dans les ressources du foyer, il semble logique de se tourner d'abord vers ce que l'on sait de la présence du livre dans les campagnes. Bien peu, en réalité : les travaux sur la diffusion du livre ont toujours privilégié les villes, laissant les villages dans l'ombre. Les études dont on dispose, fondées sur l'exploitation des inventaires après décès, donnent un ordre de grandeur très variable selon les régions. Autour de Meaux, des livres sont inventoriés chez un peu plus de $40 \%$ des paysans dans la seconde moitié du XVIII ${ }^{\mathrm{e}}$ siècle ${ }^{27}$. Autour d'Orléans, le chiffre est de $15 \%$; dans le Jura, il s'établit à $6 \%$ et il n'est pas supérieur dans le bailliage lorrain de Vézelise dans les années 1770, alors que le cadre de vie domestique s'est considérablement amélioré et que les petites écoles sont très répandues ${ }^{28}$.

À s'en tenir au contenu des inventaires après décès, la disponibilité des livres pour les enfants partant à l'école semble donc très faible, dès qu'on s'éloigne des campagnes proches de Paris. Le constat n'est pas absolument étonnant : la possession de livres reste un seuil culturel bien plus sélectif que la littératie, en ville comme à la campagne. Beaucoup d'enfants pouvaient donc se rendre à l'école les mains vides. Au pire le maître ne s'en occupait pas, au mieux les enfants disposaient des livres de l'école, lorsqu'il y en avait. Une partie des apprentissages élémentaires pouvait d'ailleurs se faire sans le truchement des livres, à l'aide des «écritures exposées » éventuellement disposées sur les murs : les tables d'alphabet et de syllabes, les sentences pieuses affichées aux murs de la classe, le grand missel qui sert aux lectures collectives et aux leçons de plain-chant. Mais moins encore pour les livres laissés aux mains des enfants que pour d'autres types de livres, on ne peut en rester à ce que révèlent les inventaires après décès. Si ce qu'on confie aux écoliers est par principe un matériel peu coûteux et peu précieux, c'est aussi ce qui, par définition, est négligé par la prisée notariale. $\mathrm{ABC}$ et petits catéchismes n'apparaissent jamais dans les inventaires après décès, alors que leur production est répandue sur tout le territoire et que les tirages atteignent des chiffres très élevés, bien supérieurs aux besoins des marchés urbains. Il faut donc inverser l'analyse et repartir de l'étude de la diffusion des livrets à bon marché, pour préciser la disponibilité de l'écrit dans les campagnes, ainsi que la nature de l'offre.

La Lorraine constitue un bon observatoire de ces phénomènes. Au XVIII ${ }^{\mathrm{e}}$ siècle, c'est dans la boutique du marchand mercier ou épicier que les populations rurales viennent faire leurs emplettes, en plus de ce qu'ils achètent auprès des colporteurs, ou sur les foires et les marchés. Les sources fiscales de la fin du XVIII ${ }^{\mathrm{e}}$ siècle suggèrent que plus d'un village lorrain sur quatre comptait au moins un commerçant à la veille de la Révolution française. Les tissus, la mercerie et la quincaillerie, un peu d'épicerie forment le fonds de la boutique, mais on y trouve aussi quelques livres, du papier et du matériel d'écriture. La quasi-totalité des marchands merciers des bourgs en proposent, ainsi qu'une petite moitié de ceux qui ont

\footnotetext{
${ }^{27}$ M. Baulant, «L'appréciation du niveau de vie. Un problème, une solution », Histoire \& Mesure, 1989, 4, p. 267-302.

${ }^{28}$ Respectivement G. Rideau, De la religion de tous à la religion de chacun. Croire et pratiquer à Orléans au XVIII ${ }^{e}$ siècle, Rennes, 2009, p. 282 ; M. Vernus, «La diffusion du petit livre de piété et de la bimbeloterie religieuse dans le Jura (XVIII ${ }^{e}$ siècle) », La diffusion du savoir de 1610 à nos jours. Actes du 105 congrès national des sociétés savantes, Paris, 1983, t. I, p.127-141; T. Volpe, «Lectures lorraines sous l'Ancien Régime : l'exemple du Saintois », Annales de l'Est, 55, 2005, p. 97-118.
} 
fixé leur commerce dans les villages ${ }^{29}$. On doit donc pouvoir trouver des livres dans une localité sur dix, sans même devoir compter sur les ressources du commerce itinérant et périodique - ce qui, compte tenu de la densité de l'occupation humaine en Lorraine, en fait une marchandise relativement accessible. En fait d'offre de librairie, il s'agit le plus souvent de quelques volumes de piété : des psautiers et des heures, des alphabets et des catéchismes; peu de titres, mais en abondance et à faible coût, de quoi fournir tout un village. Le marchand mercier du village de Faulquemont, Joseph Coster, aligne au moment de sa faillite en 1743 « 1 cent de abc pour 1.101 . et 9 sicvillitté et noé pour 11 . ${ }^{30}$. Chez Léopold Réalle, marchand à Mairy, dix douzaines et demi d'ABC forment toute l'offre de librairie en $1780^{31}$. Chez la grande majorité des boutiquiers, l'offre restreinte se justifie par le profil des consommateurs locaux, relativement moins alphabétisés que dans les villes et relativement moins enclins aux achats de commodités, à niveau social équivalent ${ }^{32}$. Mais elle résulte aussi du cadre fixé par la législation royale, même si les contraventions ne sont pas rares. D'après les dispositions du Code de la librairie de 1723, élargies en 1744 à l'ensemble du royaume, ces boutiquiers ne peuvent vendre que « des A.B.C., almanachs et petits livres d'heures et de prières ", de manière à satisfaire les besoins élémentaires des écoliers et des fidèles. En autorisant ce commerce aux non-libraires, la monarchie met en place les conditions de possibilité d'une offre de librairie, présente de manière relativement capillaire sur le territoire et dont les formes rappellent celles qu'elle aura encore tout au long du XIX ${ }^{\mathrm{e}}$ siècle, voire plus $\operatorname{tard}^{33}$. En l'absence de toute politique scolaire, cette disponibilité des livres à bon marché constitue une contribution fondamentale de l'État royal au fonctionnement des petites écoles.

Les inventaires des boutiques rurales permettent d'enrichir notre compréhension de la présence de la culture écrite au village. Ils montrent que le petit livre scolaire et religieux à moins de 5 sous est une denrée courante dans les campagnes, au moins celles du nord-est de la France. Les quantités proposées permettent également de penser que ces brochures étaient plus présentes dans les foyers - et présentes dans un plus grand nombre de foyers - que ne l'enregistrent les inventaires après décès. En ce sens, la proportion d'enfants qui allaient à l'école sans livre était probablement moins importante qu'on ne l'imagine. Ces boutiques comptent, pour notre propos, pour ce qu'elles offrent, mais également pour la manière dont elles l'offrent. Les travaux que les sociologues ont consacrés aux « faibles lecteurs » aident à penser la manière dont l'appareil commercial des campagnes a facilité la pénétration de l'imprimé dans les intérieurs populaires. On sait que les lecteurs les moins familiers de la culture écrite acquièrent le plus souvent le livre là où ils regroupent leurs pratiques générales de consommation. Mêlé aux rubans, aux épingles et aux boutons, exposé en ligne et en série comme les couteaux ou les plumes, le livre n'est pas un objet intimidant : il est maintenu dans sa matérialité de produit de consommation. S'il est là, c'est que son achat entre dans l'ordre du pensable, du possible, voire du souhaitable. Les dimensions restreintes de l'offre limitent par ailleurs les doutes et les hésitations qui naîtraient de propositions plus variées. D'une certaine manière, « c'est plutôt du livre qu'on achète, qu'un livre particulier ${ }^{34}$.

\footnotetext{
${ }^{29}$ J. Villain, «La boutique et les différentiels intra-régionaux de l'offre : le cas de la Lorraine à la fin du XVIII ${ }^{\mathrm{e}}$ siècle », Il commercio al minuto. Domanda e offerta tra economia formale e informale. Secc. XIII-XVIII, Florence, 2015, p. 185-212.

${ }^{30}$ A.D. Meurthe-et-Moselle, 49 B 163, inventaire du 18 avril 1743.

${ }^{31}$ A.D. Meurthe-et-Moselle, 49 B 218.

${ }^{32} \mathrm{~J}$. Villain, Appareil commercial et diffusion des biens de consommation au XVIII ${ }^{e}$ siècle. Aires et structures du commerce des commodités en Lorraine centrale et méridionale, années 1690-1791, Thèse, Université Paris-I, 2015 , p. 381.

${ }^{33}$ F. Barbier, « La diffusion de l'imprimerie en Eure-et-Loir au XIX ${ }^{\mathrm{e}}$ siècle », J.-Y. Mollier (dir.), Le commerce de la librairie en France au XIX siècle (1789-1914), Paris, 1997, p. 151-168.

${ }^{34} \mathrm{~J}$. Bahloul, Lectures précaires. Études sociologiques sur les faibles lecteurs, Paris, 1990.
} 


\section{Des livres pour aller à l'école}

Une fois précisée la disponibilité des livres, le problème reste entier de savoir quels ouvrages les enfants apportaient dans les écoles. Ces expériences ordinaires, rendues routinières, réglées par le sens pratique, ont laissé peu de traces. Elles n'ont été que très rarement réfractées par des opérations productrices de matériel écrit, judiciaires ou patrimoniales. Lorsqu'elles le font, elles les donnent à voir latéralement, en les pliant à leur logique. Ainsi, les inventaires d'écoles rurales dressés au début de la Révolution ne décrivent précisément que les livres qui appartiennent à l'institution et laissent de côté ceux des enfants, qui pourraient être réclamés par des particuliers. L'inventaire de l'école d'Ay, gouvernée par les filles de la communauté de l'Enfant Jésus, dressé le 21 mai 1792 après le départ des sœurs, montre deux salles de classe avec leurs bancs, les cartes d'instruction destinées aux plus jeunes, les tables à écrire pour les plus grandes, deux tableaux et « une petite armoire aussi bois de chêne à côté servant à y renfermer les livres des enfants, dans lesquels se sont trouvés plusieurs volumes à l'usage des enfants et qui nous a paru leur appartenir », et qui ne sont donc pas inventoriés ${ }^{35}$. Les enseignements de ces inventaires doivent être confrontés à ce que l'on sait des petites possessions enfantines, telles que les révèlent par exemple les procès-verbaux de levées de cadavres, ou les gages pris sur les responsables d'infractions rurales, enfant surpris à chaparder dans les jardins ou à laisser divaguer son troupeau dans les champs ${ }^{36}$. Rien dans tout cela n'atteste que ces livres étaient lus, encore moins qu'ils étaient utilisés à l'école, mais ces éclairages attestent une relation de familiarité de l'enfant avec un livre qui est «à lui ». La rareté des exemplaires conservés permet mal d'appréhender les opérations d'appropriation liées à ces petites possessions. Le relevé des mentions manuscrites sur les livrets de la Bibliothèque bleue de Troyes met en évidence des marques de possession enfantines à partir de la seconde moitié du XVIII ${ }^{\mathrm{e}}$ siècle, sur des Civilités ou des Règles de bienséance très certainement utilisées dans les classes ${ }^{37}$.

À la différence des indices pris dans le filet des productions administratives, la mise en récit des expériences d'enfance fournit à l'historien un matériau fascinant de cohérence. Les souvenirs recomposés que Nicolas Rétif de La Bretonne livre de sa formation scolaire et de ses lectures de jeunesse, dans la Bourgogne des années 1740, constituent un témoignage « exceptionnel normal », unique mais susceptible d'éclairer des phénomènes sociaux diffus ${ }^{38}$. Fils d'un laboureur aisé, Rétif appartient à la couche supérieure de la paysannerie bourguignonne. Quoique son auteur ait souvent fréquenté l'école, Monsieur Nicolas se présente comme le récit d'une autodidaxie, d'une trajectoire réfractaire aux cadres établis, dans laquelle les connaissances transmises par les maîtres comptent moins que l'élan qui pousse le jeune Nicolas à «voler le feu », à s'emparer des outils de la lecture. L'institution scolaire marque pourtant tout le récit des premières années, sous la forme d'une ombre portée, à travers les gestes et les pratiques inculquées qui modèlent le rapport au livre du jeune enfant, y compris lorsque celui-ci se représente comme le maître de ses apprentissages.

La «bibliothèque » personnelle du petit Rétif compte tout d'abord un ensemble de livres utilisés dans le cadre scolaire : un syllabaire latin tout élimé, sur lequel il apprend à épeler et à déchiffrer les mots à l'école de maître Jacques, et un psautier latin-français dont il se sert sans doute à l'école pour lire le latin et qu'il utilise pour " jouer à la messe » avec ses frères

\footnotetext{
${ }^{35}$ A.D. Marne, E dép. 2483.

${ }^{36}$ F. Bayard, « Au cœur de l'intime : les poches des cadavres. Lyon, Lyonnais, Beaujolais, XVII - XVIII $^{\mathrm{e}}$ siècle », Bulletin du centre d'histoire économique et sociale de la région lyonnaise, 2, 1989, p. 5-41; P. Martin, Une religion des livres (1640-1850), Paris, 2003, p. 531-532.

${ }^{37}$ M.-D. Leclerc, « Ex-libris manuscrits et notes dans les impressions de grande diffusion $\left(\mathrm{XVII}^{\mathrm{e}}-\mathrm{XIX}^{\mathrm{e}}\right.$ siècle) », Histoire et Civilisation du livre, 2, 2006, p. 323-345.

${ }^{38}$ N. Rétif de la Bretonne, Monsieur Nicolas, éd. P. Testud, Paris, 1989.
} 
et sœurs. Dans ses usages ludiques, le psautier devient l'accessoire d'un jeu de rôles, qui rappelle celui qu'endosse le maître pour enseigner aux enfants le service de l'église. Ce psautier est de la famille des petits livres pieux qu'on trouve dans les poches des enfants : Rétif raconte qu'il l'emporte avec lui dans ses moments libres, dans les champs ou au jardin, et qu'il s'isole sur un vieux pommier pour le lire tranquillement. C'est dans ce livre qu'il situe sa première « révélation littéraire ". Alors qu'il a ouvert son livre à la page du psaume Super flumina Babylonis, la beauté de la langue française lui apparaît brusquement. Rétif raconte qu'il est « tombé » sur le psaume «à l'ouverture du livre », ce qui laisse penser qu'il pratiquait, avec son psautier, des tirages au sort durablement attestés dans la société rurale, malgré les condamnations ecclésiastiques ${ }^{39}$. Sa bibliothèque personnelle compte également les Méditations du père jésuite Jean Busée «qu'on me sacrifiait», dans un geste probablement représentatif des arbitrages familiaux qui désignent un livre, pas le plus coûteux ni le plus récent (la première édition du texte de Busée remonte à 1612), qu'on laissera maltraiter par l'enfant dans ses exercices de lecture. Sa mère lui donne enfin une Vie de Jésus-Christ in- $4^{\circ}$, dont il apprécie les «belles marges » et les «jolies lettres frisées ». Il le manipule comme un livre liturgique, en le plaçant sur deux bâtons plantés dans le mur en guise de lutrin, pour chanter sur le ton des épîtres les versets latins imprimés dans les marges de l'ouvrage. Comme pour le simulacre de messe, Rétif reproduit probablement des manières de manipuler le livre et des exercices pratiqués en classe, qui forment la matrice de son rapport à l'écrit dans ses moments de loisir.

Un syllabaire, un psautier, un livre de dévotion périmé : voilà sans doute ce qui forme le paquet de livres du jeune Rétif dans ses premières années d'école. Redisons-le : on est ici dans un milieu de paysannerie aisée, dont les pratiques ne peuvent être généralisées - trois ou quatre livres pour un seul enfant! Les éléments rassemblés peuvent malgré tout être rapprochés des propos des correspondants de Grégoire, qui décrivent un matériel tout aussi limité. Ce que les enfants apportent à l'école sont des syllabaires, le catéchisme, le psautier et les Heures du diocèse ; de petits livres de dévotion, l'Instruction de la jeunesse, le Chemin du ciel, des vies de saints; des petits livres de civilité. Les correspondants de Grégoire sont-ils passés à côté des réalités scolaires ou les petites écoles constituent-elles vraiment un monde imperméable aux productions profanes ? La question des livres assignés à l'enfant revient à poser celle de l'adhésion des parents à une "culture sociale de l'école », qu'il faut maintenant s'attacher à décrire.

\section{Ce que fabrique l'école}

Le dernier maillon de cette production sociale des livres scolaires est l'école elle-même. La manière dont on conçoit les étapes de l'apprentissage, les méthodes d'enseignement, le cadre matériel même de l'école, développent un certain rapport à la lecture, aux textes et à l'objet-livre, autrement dit une culture scolaire de l'écrit qui participe aux arbitrages des acteurs.

\section{Des expériences de littératie.}

D'un bout à l'autre de l'Europe, pendant toute l'Époque moderne et encore longtemps au $\mathrm{XIX}^{\mathrm{e}}$ siècle, la dimension orale des matériaux écrits reste au cœur des processus par lesquels les enfants arrivent à les reconnaître ${ }^{40}$. Dans ses premières étapes, l'alphabétisation

\footnotetext{
${ }^{39}$ H.J. Martin, Une religion des livres..., p. 526-528. Le tirage au sort consiste à ouvrir un livre au hasard; la première phrase lue est réputée avoir une valeur prophétique.

40 De très nombreux travaux ont insisté sur ce point. Voir par exemple A. Messerli, Lesen und Schreiben, 1700 bis 1900. Untersuchung zur Durchsetzung der Literalität in der Schweiz, Tübingen, 2002, p. 255-258.
} 
s'apparente à une opération de reconnaissance : apprendre à lire, c'est apprendre à donner voix à un texte en mobilisant les ressources de la mémoire. C'est faute d'avoir activé ce capital que Rétif de La Bretonne reçoit le fouet de son maître. Sur son syllabaire élimé par le frottement des pouces ou composé avec un matériel défectueux, le tuum du Pater noster est devenu $t u$ in : c'est ce qu'il s'obstine à lire, alors que sa sœur et tous ses camarades lui soufflent la bonne leçon, qu'ils connaissent par cœur et qu'ils savent retrouver sur leur livre. Par la suite, l'adolescent fait un usage plus efficace de cette mémoire auditive. À onze ans, alors qu'il sait déchiffrer le latin, il invente un subterfuge pour apprendre à lire seul le français. Il met sa sœur aînée au défi de lire en français sa Vie de Jésus Christ, aussi bien qu'il peut le faire en latin: «je la suivais des yeux et mon excellente mémoire me fit tout retenir mot pour mot. Dès qu'elle se fut éloignée, je repris la page et la déchiffrai, non sans admiration ». Il réitère l'opération sur la Bible de son père, "dont je savais par cœur tout l'historique, que je lus couramment. Ainsi, je savais lire le français $»{ }^{41}$.

Dans les deux cas, la réussite de l'opération suppose que l'enfant soit mis au travail sur des textes qui appartiennent à son univers sonore familier. Anne-Marie Chartier souligne combien la primauté de l'oralisation s'accorde avec la nature des textes sur lesquels les enfants travaillent, qui sont le plus souvent des textes performatifs: prières, actes de contrition, mais également actes notariés qui font l'objet d'une lecture à haute voix avant signature $^{42}$. On doit à Xavier Bisaro d'avoir mis en lumière une autre dimension jusqu'alors négligée par l'historiographie, celle de la contamination des formes de la lecture orale par l'omniprésence du chant dans le quotidien scolaire ${ }^{43}$. Dans ses premières étapes, l'apprentissage de la lecture dans l'école d'Ancien Régime est donc étroitement lié aux supports utilisés. De manière significative, de nombreux contrats d'embauche de maîtres ne rapportent pas les tarifs d'écolage à des compétences abstraites (savoir épeler, savoir lire, savoir compter), mais à des textes particuliers. Celui de Claude Villain, nommé à VillersMarmery (Marne) en 1763, stipule que les habitants s'obligent « de lui payer par chaque mois d'enfants qui seront à l'alphabet, deux sols six deniers ; pour ceux qui seront aux Septiaux [sept psaumes pénitentiaux] et aux Matines, trois sols ; pour ceux qui liront dans la lettre, la civilité et écriront, quatre sols ; et le même prix pour ceux qui apprendront l'arithmétique et chanter ${ }^{44}$. Ce type de document, relativement courant, est généralement utilisé pour illustrer l'émiettement des compétences et la progression individuelle des apprentissages. Mais il témoigne également d'une culture villageoise de l'école qui définit ce que l'on attend des enfants en termes de capacités pratiques (dire les psaumes, suivre l'office des matines, comprendre le contenu d'un contrat). La fonction de ces mentions n'est pas tout à fait claire : renvoie-t-elle aux ouvrages effectivement fournis par les parents et utilisés par les enfants? Fonctionne-t-elle comme un programme de lectures suggéré par le maître ou comme simple métonymie des compétences à acquérir ? Des éléments d'interprétation sont sans doute à trouver dans les termes du contrat, qu'ils aient été fixés par le maître ou qu'ils résultent d'un canevas réutilisé par la communauté à chaque embauche de maître. Dans tous les cas, la trame tarifaire rappelle que les compétences de lecture sont indissociables de la manipulation des objets, de sorte que le choix du livre de classe est déjà une projection des savoir-faire dont on souhaite l'acquisition par l'enfant.

\footnotetext{
${ }^{41}$ N. Rétif de la Bretonne, Monsieur Nicolas..., p. 80.

42 A.-M. Chartier, «Faire lire les débutants : comparaison de manuels français et américains (1750-1950)», Histoire de l'éducation, 138, 2013, p. 35-68, p. 38.

${ }^{43}$ X. Bisaro, Chanter toujours. Plain-chant et religion villageoise dans la France moderne (XVI ${ }^{e}$ XIX $X^{e}$ siècle), Rennes, 2010 ; id., «La voix des pauvres : chant et civilité oratoire dans les écoles de charité de Lyon à la fin du XVII ${ }^{\mathrm{e}}$ siècle », Histoire de l'éducation, à paraître.

${ }^{44}$ Cité dans H. Jadart, Les Écoles de Reims et de son arrondissement en 1774, Reims, 1888, p. 57.
} 
Que le livre porte en lui une préfiguration de ses usages ne le réduit pas pour autant à sa fonction instrumentale. Les attentes des parents qui remettent un psautier à leur enfant partant pour l'école ne se bornent pas toujours aux frontières de la littératie chrétienne : que l'on puisse améliorer ses conditions de vie ou l'avenir de sa progéniture par la maîtrise de la lecture et de l'écriture fait l'objet d'une croyance assez largement partagée dans les milieux les moins défavorisés. Les compétences acquises sont destinées à circuler vers d'autres espaces, vers la maison ou vers l'église, où leur articulation à d'autres matériaux ou à d'autres pratiques de la culture écrite peut contribuer à en développer les potentialités. Les compétences inculquées à l'enfant pendant une scolarité parfois très brève seront d'autant mieux consolidées qu'elles trouveront à s'appliquer - à défaut de livres - aux papiers qui s'accumulent au XVIII ${ }^{\mathrm{e}}$ siècle dans les foyers, même dans les intérieurs paysans. Jean Borion, régent à Bois, conseille ainsi à maître Bernard, laboureur à bœufs de la paroisse de Saint-Genis-de-Saintonge, dans les années 1760, de faire travailler son fils sur les papiers de famille pour soutenir ses apprentissages scolaires : « $J$ eay L'honneur de vous priyer de donner l'œil à votre fils qu'il écrive et lize fortement dans les actes. Il faut que vous y portiez vos soins. Il y a de la peine pour savoir son nécessaire... ${ }^{45}$. Il semble qu'on pourrait appliquer aux sociétés modernes la suggestion de Bernard Lahire, qui invite à rendre raison des succès scolaires en milieu populaire, en les rapportant aux formes familiales de la culture écrite, à la présence d'écritures domestiques manipulées, suscitées et organisées, au moins autant qu'au capital scolaire transmis par les parents ${ }^{46}$.

Le livre véhicule ainsi un imaginaire des possibles sociaux qui peuvent faire du moment de son achat, un véritable rite de passage. Pour l'enfant qui commence à bien déchiffrer, ce sont les Heures du diocèse dont on a vu qu'elles l'accompagnaient ensuite au quotidien. Dans les familles aux aspirations sociales plus soutenues, c'est l'achat du rudiment qui marque les débuts de l'apprentissage du latin, la voie ouverte vers le collège et, espère-t-on, la prêtrise. Dans leur réponse à l'abbé Grégoire, les Amis de la Constitution d'Auch évoquent l'événement que constitue, pour un enfant des milieux populaires ruraux en quête d'ascension sociale, le premier « livre à soi » :

Le jeune homme allant à l'école chez le curé, qui le faisait lire une fois par mois au plus, quoique les pauvres parents se privassent journellement des petits services que leur aurait rendus leur enfant ; ce jeune homme, disons-nous, n'était pas plus tôt parvenu à la possession des Heures du diocèse, qu'il les avait constamment dans sa poche au travail, et dans les mains au moment du repos. Il lisait toute sa vie, et mourait sans avoir jamais su lire ${ }^{47}$.

Formidable et terrible expression de cette littératie restreinte, que Jean Quéniart proposait d'appeler «semi-alphabétisation ${ }^{48}$ - mais aussi témoignage de la force des gestes incorporés par la fréquentation scolaire, même minimale, des jeunes ruraux.

\section{Manières d'enseigner}

Les expériences de lecture à l'école sont aussi étroitement liées aux manières d'enseigner. La longévité des méthodes individuelles est souvent imputée à la multiplicité des supports de lecture, qui rend impossible, jusque tard dans le $\mathrm{XIX}^{\mathrm{e}}$ siècle dans certaines zones rurales, la mise en place de méthodes simultanées. L'émiettement de la fréquentation scolaire, les arrivées qui s'échelonnent tout au long de l'année finissent de compliquer les dynamiques de

\footnotetext{
${ }^{45}$ L. Robin, Petites écoles et leurs régents en Saintonge et en Aunis avant la Révolution, 1685-1789, La Rochelle, 1968, p. 132.

${ }^{46}$ B. Lahire, Tableaux de famille : heurs et malheurs scolaires en milieux populaires, Paris, 2012.

${ }^{47}$ Réponse des Amis de la Constitution d'Auch, dans A. Gazier, Lettres à Grégoire..., p. 98.

${ }^{48} \mathrm{~J}$. Queniart, Culture et société urbaine dans la France de l'Ouest au XVIII 'e siècle, Paris, 1978.
} 
groupe. Les progrès de l'enseignement simultané sont pourtant notables au XVIII ${ }^{\mathrm{e}}$ siècle. Après les congrégations enseignantes dans la seconde moitié du XVII siècle, un certain nombre d'évêques encourage à la nouvelle méthode, dont le succès rencontré dans les villes par les Frères des écoles chrétiennes favorise enfin la diffusion ${ }^{49}$. Le contrat d'embauche du régent de Montigny-le-Roi (Haute-Marne), en 1788, stipule qu'il devra aller passer une huitaine de jours à l'école des frères de Langres, pour y apprendre la méthode «d'enseignement qui s'y pratique ${ }^{50}$. En 1790 , le correspondant de Commercy signale à l'abbé Grégoire que dans les campagnes qui environnent la ville, " chaque enfant a un livre différent, ce qui empêche les maîtres d'école de suivre pour enseigner la méthode des Frères de la doctrine chrétienne qui paraît être la meilleure $\gg{ }^{51}$.

Dans tous les cas, il n'est pas de bonne méthode d'opposer l'absolue hétérogénéité des supports qui règnerait dans les petites écoles, et la parfaite normalisation à laquelle tendent, par exemple, les Frères des Écoles chrétiennes. Là où l'enseignement simultané est attesté, on sait mal comment le maître a procédé pour obtenir des parents que tous les enfants utilisent le même livre. À l'inverse, la diversité des instruments n'est pas forcément un obstacle. Les prières élémentaires comme le Pater Noster se trouvent dans les différents types d'ABC. Il faut également tenir compte de l'effet de lissage produit par l'étroitesse de l'offre de livres chez les marchands locaux et de la force de persuasion du maître, dont certains devaient être capables d'orienter les choix des parents. Il n'est enfin pas exclu que les régents aient fait commerce des ouvrages de classe, même si cela était théoriquement interdit et si les traces que l'on en trouve sont rares. Ces éléments ont d'autant plus de poids qu'ils sont relayés, au $\mathrm{XVIII}^{\mathrm{e}}$ siècle, par un discret mouvement de fond, celui de l'institutionnalisation de la fourniture des livres scolaires.

De manière plus ou moins formalisée, sous des statuts divers, des livres sont en effet là, dans l'école. Ils installent une culture scolaire de l'écrit chez des enfants que leur environnement familial ne prépare pas à cette familiarité, et permettent éventuellement des temps d'enseignement simultané. Il n'est pas rare que le curé lègue quelques ouvrages à l'école paroissiale ou que la fabrique cède de vieux livres d'office, en particulier des graduels ou des missels qui servent à apprendre à chanter aux enfants et que leur grande taille rend particulièrement commodes pour un usage commun. Il y a aussi les livres personnels du maître. Gyrovague ou installé, le maître peut disposer de ses propres outils de travail, qu'il accepte de mettre à disposition d'une partie des élèves, les plus pauvres, les plus méritants ou les plus avancés.

La question des fournitures scolaires est progressivement transformée, au cours du XVIII ${ }^{\mathrm{e}}$ siècle, par la multiplication des fondations pieuses qui institutionnalisent l'approvisionnement des enfants en livres d'école. En plus de constituer une rente pour rémunérer le maître ou la maîtresse, comme cela se pratique depuis le siècle précédent, certains donateurs introduisent un nouveau type de clause : la fourniture des supports scolaires, papier, encre, plumes et livres, pour les écoliers nécessiteux. Ces distributions ne sont pas un phénomène nouveau (on en trouve des témoignages dès la fin $\mathrm{du} \mathrm{XVI}^{\mathrm{e}}$ siècle dans les institutions hospitalières des grandes villes), mais elles profitent désormais plus souvent aux écoles de campagne. C'est peut-être ces dispositifs que l'abbé Grégoire a en tête lorsqu'il pose la question de l'uniformité des livres de classe dans les villages. Une cinquantaine de fondations a, pour l'instant, été repérée mais le chantier reste ouvert. Les modalités matérielles en sont

\footnotetext{
${ }^{49}$ Lettre pastorale de monseigneur l'évêque de Bayeux touchant les petites écoles. Avec la méthode pour apprendre en peu de temps à lire, écrire, faire le catéchisme et chanter, Caen, $1690 \mathrm{ou}$, plus tardif, Abrégé des statuts du diocèse de Chaalons, Châlons, 1770.

${ }^{50}$ Cité dans M. Fayet, Recherches historiques et statistiques sur les communes et les écoles de la Haute-Marne, Langres-Paris, 1879, p. 77.

${ }^{51}$ B.N., NAF 2798 , fol. 25 [s.d.].
} 
extrêmement diverses (montant affecté, mode de gestion envisagé, destination des ouvrages qui peuvent revenir aux enfants ou constituer un fonds appartenant à l'école). Ce qui ne varie pas, en revanche, est l'ordre des livres : alphabets, catéchismes, épîtres et évangiles, Pensées chrétiennes et Sages entretiens, petites Heures, vies de Jésus-Christ, histoires de l'Ancien et du Nouveau Testament constituent toute la bibliothèque scolaire envisagée par les donateurs ${ }^{52}$.

Les petites écoles de l'Ancien Régime ne sont pas le royaume de la fantaisie. Les injonctions ecclésiastiques et les fonds des boutiques de village convergent pour désigner un ordre des livres relativement cohérent. Il est possible que la force de ce consensus incorporé dans toute une série de dispositifs matériels - fondations scolaires, contrats d'embauche des maîtres - ait provoqué un effet de masque et rendu invisible d'autres réalités. Que les témoignages manquent sur l'utilisation des romans profanes, des almanachs et des gazettes dans les écoles du XVIII ${ }^{\mathrm{e}}$ siècle ne semble ainsi pas de nature à clore tout à fait le dossier.

Si cette librairie scolaire est encore principalement faite du tout-venant des livres de piété de grande circulation, la tendance à infantiliser les supports utilisés en classe se dessine fortement. Engagée par l'Église à la fin du XVII ${ }^{\mathrm{e}}$ siècle, elle s'appuie sur le souci d'une plus grande efficacité dans la transmission des savoirs catéchétiques, susceptible de remédier la société toute entière. C'est bien ce qu'envisagent aussi les rédacteurs des cahiers de doléances qui demandent en 1789, non un catéchisme religieux, ni un catéchisme économique, mais bien un catéchisme civique, "lesquel sera enseigné dans les paroisses et dont l'usage constant attachera dans peu d'années les Français à la loi par le lien indissoluble d'une éducation nationale ${ }^{53}$.

\footnotetext{
${ }^{52}$ Voir par exemple P. Dentin, «Fondation d'une école de pauvres enfants à Bourseville en 1706 », Bulletin de la Société d'émulation d'Abbeville, 24, 1977, p. 239-243.

${ }^{53}$ E. Allain, La question d'enseignement en 1789 d'après les cahiers de doléances, Paris, 1886.
} 